\title{
Scale-selective Turbulence Reduction in H-mode Plasmas in the TJ-II Stellarator
}

\author{
T. Happel ${ }^{1}{ }^{*}$ T. Estrada, E. Blanco, C. Hidalgo, G. D. Conway ${ }^{1}$, U. Stroth ${ }^{1}$, and the TJ-II Team \\ Laboratorio Nacional de Fusión, Association Euratom-Ciemat, 28040 Madrid, Spain and \\ ${ }^{1}$ Max-Planck-Institut für Plasmaphysik, Association Euratom-IPP, 85748 Garching, Germany
}

\begin{abstract}
Wavenumber spectra of density turbulence in L- and H-mode plasmas have been measured in the TJ-II stellarator by means of Doppler reflectometry. A pronounced suppression of the density fluctuation level is observed in $\mathrm{H}$-mode close to the radial position of maximum radial electric field $\left(E_{r}\right)$ shear. Furthermore, intermediate scale density turbulence is reduced preferentially. This effect can be interpreted within the framework of vortex stretching feeding energy through Reynolds stress into zonal flows, while shear decorrelation of turbulent structures might not play a central role in TJ-II. Moreover, it is shown that in both L- and H-mode, the phase velocity of density fluctuations does not depend on the structure scale.
\end{abstract}

PACS numbers: 52.35.Ra, 52.55.Hc, 52.70.Gw, 52.25.Xz

\section{INTRODUCTION}

Since more than a quarter century the H-mode plasma regime [1], characterized by a bifurcation-type transition to improved confinement and reduced turbulence level, is known. Although significant progress has been made in describing the dynamics of the transition, the physical mechanism triggering the $\mathrm{H}$-mode has still not been clearly identified. The reduced turbulence levels in $\mathrm{H}-$ modes are normally accompanied by strong radial electric field $\left(E_{r}\right)$ shears [2-6]. However, how exactly the interplay between $E_{r}$ shear and turbulence is, has also not been fully clarified yet.

Shear decorrelation has been put forward to explain reduced transport by the tearing apart of turbulent eddies [7]. For the shear decorrelation mechanism to be effective, the so-called BDT-criterion [7] must be fulfilled (for a review, see [8]), which states that if the poloidal plasma velocity shear (e.g. due to a shear in $\left.E_{r}\right)$ is strong enough compared to the structure scale and turbulence growth rate, the structure can be torn apart, reducing its radial extent. Shear decorrelation is most pronounced if characteristic structures are radially elongated and poloidally small. In the DIII-D tokamak, beam emission spectroscopy data from an L-mode plasma shows signatures of shear decorrelation [9].

It is argued that the physical mechanism responsible for the L-H transition might be the zonal flow (ZF), which is fed by the turbulence itself through Reynolds stress [10-12]. If fluctuations are isotropic, the Reynolds stress is zero. However, if vortices are tilted by sheared flows, they become non-isotropic and thus an energy transfer channel from the turbulence into the ZF is established, decreasing the turbulence energy. More recently, it has been observed both in experiment [12] and simulation [13] that predominantly small scales feed energy into the (large scale) ZF, and it has been argued that a

*Electronic address: tim.happel@ipp.mpg.de tilting and stretching out of turbulent structures can be sufficient to reduce the turbulence energy [12-15].

Scale-resolved turbulence studies are necessary in order to understand the underlying physics processes and their potential interactions [16, 17]. First wavenumber spectra were measured in the mid-70's with electromagnetic wave (infrared and microwave) scattering techniques $[18,19]$, followed up by measurements on a variety of machines [20-23]. Doppler reflectometry and far infrared scattering have been employed on the Tore Supra tokamak to identify turbulence scales and investigate the collisionality dependence of density turbulence spectra [24-27]. However, as of today, the scale-dependence of the turbulence reduction in H-mode plasmas has not been addressed thoroughly, although preliminary work has been presented [28-30]. Furthermore, the importance of the investigation of small-scale turbulence has gained increased attention due to the prediction of high anomalous transport by electron temperature gradient (ETG) turbulence [31]. In particular, recently investigations of a large wavenumber range have been conducted in the DIII-D tokamak in order to identify turbulence mechanisms in plasma scenarios with dominant electron cyclotron resonance heating (ECRH) [32].

Experiments in the TJ-II stellarator [33] have shown that, at the L-H transition, $E_{r}$ becomes more negative and a pronounced $E_{r}$ shear develops together with an abrupt reduction in plasma turbulence close to the radial position of maximum $E_{r}$ shear [34]. The time evolution of both, $E_{r}$ shear and density fluctuations, indicates that the reduction in density fluctuations precedes the increase in the mean $E_{r}$ shear but is simultaneous with the increase in the low frequency oscillating sheared flow [35]. These observations, together with the amplification of long-range correlations in the potential fluctuations [36], are consistent with L-H transition models predicting plasma bifurcations triggered by ZFs [10, 11]. Further experimental evidence supporting the central role of ZFs has been recently observed in experiments carried out close to the transition threshold conditions: a coupling between flows and turbulence is measured which reveals a characteristic predator-prey 


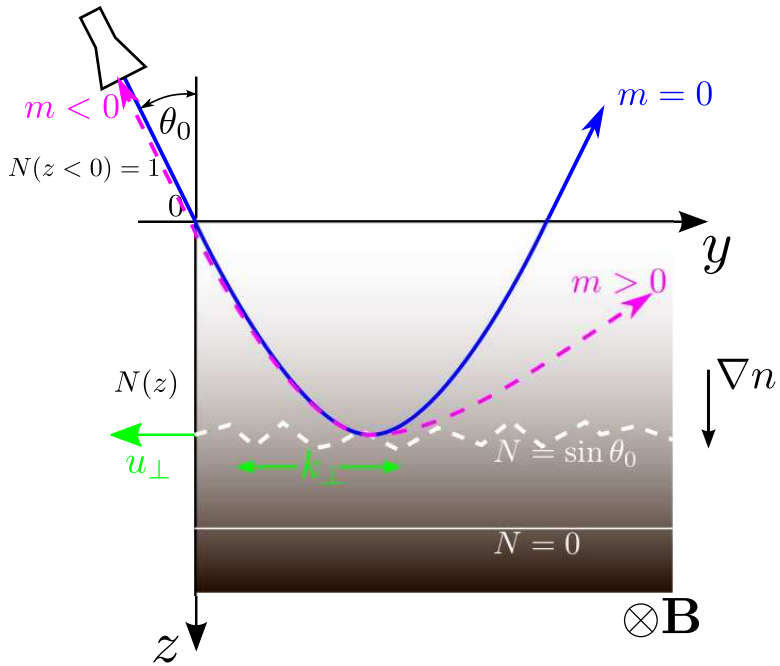

FIG. 1: Schematic drawing of oblique incidence of a microwave beam in a slab plasma with a corrugated cutoff layer.

behaviour [37].

In this paper we report on the scale-selective reduction of turbulence close to the transport barrier in H-mode plasmas of the TJ-II stellarator. The employed diagnostic technique is Doppler reflectometry, which is relatively new ( $\sim 10$ years), but used on several tokamaks and stellarators worldwide [38-44].

This paper is organized as follows: section II introduces the TJ-II Doppler reflectometer and the measurement technique, section III presents radial electric field profiles and analyzes the scale-resolved turbulence reduction, while section IV discusses the findings and presents conclusions.

\section{DOPPLER REFLECTOMETRY}

Figure 1 shows the principle of Doppler reflectometry in slab geometry. A microwave beam is launched under the angle $\theta_{0}$ with respect to the normal of the plasma vacuum surface. If the cutoff layer is not smooth but corrugated, due to turbulent density fluctuations, the microwave beam is scattered at the cutoff layer. The antenna is used as a transceiver thus that it selects the Bragg backscattering in the order $m=-1$. The intensity of the wave backscattered under $\theta_{0}$ depends on the spectral Fourier component of the density fluctuations with a wave vector perpendicular to the magnetic field $k_{\perp}$ following Bragg's law

$$
k_{\perp}=2 k_{0} \sin \theta_{0},
$$

where $k_{0}$ is the microwave vacuum wavenumber. In addition, the Doppler shift $f_{\mathrm{D}}$ of the spectrum of the backscattered signal is related to the velocity of density fluctuations $u_{\perp}$ and their perpendicular wavenumber $k_{\perp}$

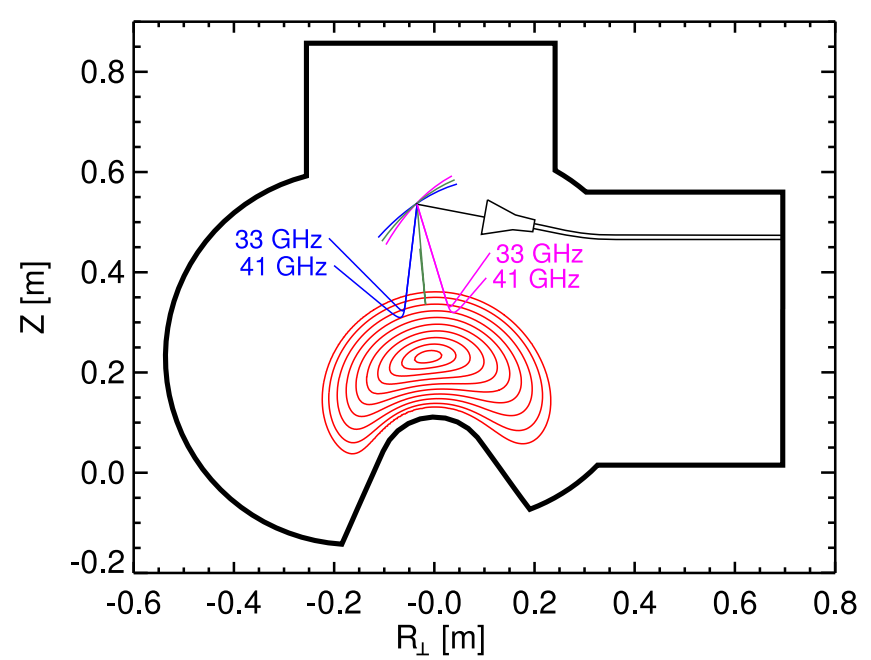

FIG. 2: Schematic of the TJ-II Doppler reflectometer. For details refer to Ref. [43].

through $f_{\mathrm{D}}=u_{\perp} k_{\perp} / 2 \pi$. Hence with the measurement of the Doppler shift $f_{\mathrm{D}}$ and the knowledge of the perpendicular wavenumber $k_{\perp}$, the perpendicular velocity of the plasma can be calculated. The formula for the plasma slab (1) is not valid for plasmas with curved cutoff-layers as for most confined plasmas. In these cases ray or beam tracing has to be used to calculate both radial position of backscattering and probed turbulence wavenumber $k_{\perp}$.

The perpendicular velocity $u_{\perp}$ is composed out of the plasma background $E \times B$ velocity $v_{E \times B}$ and the phase velocity of turbulent fluctuations $v_{\mathrm{ph}}$ :

$$
u_{\perp}=v_{E \times B}+v_{\mathrm{ph}} .
$$

When the phase velocity of turbulence is small compared to the $E \times B$ velocity, $v_{\mathrm{ph}} \ll v_{E \times B}$, the radial electric field can be deduced from $E_{r}=u_{\perp} B$, where $B$ is the magnetic field strength. Comparison of $E_{r}$ from Doppler reflectometry with $E_{r}$ obtained with a heavy ion beam probe at TJ-II showed good agreement [35]. Furthermore, comparisons with $E_{r}$ from spectroscopy in W7-AS [40] and with $E_{r}$ from a Ball pen probe in ASDEX Upgrade [45] have also shown that $v_{\mathrm{ph}} \ll v_{E \times B}$ holds. In DIII-D, $E_{r}$ from beam emission spectroscopy and charge exchange recombination spectroscopy measurements also confirm the above statement [9].

In TJ-II, a Doppler reflectometer is installed [43] which can operate in the whole range of magnetic configurations available, and in different plasma scenarios. A sketch of the system is shown in Fig. 2. The possibility to steer the beam with the ellipsoidal mirror allows for the measurement of density turbulence scales between $k_{\perp}=3$ and $15 \mathrm{~cm}^{-1}$. Although the $u_{\perp}$ measurement with Doppler reflectometry is rather straightforward, the investigation and reliability of wavenumber spectrum measurements depend particularly on the spectral resolution of the Doppler reflectometer [46-49]. During the design of the TJ-II Doppler reflectometer, partic- 


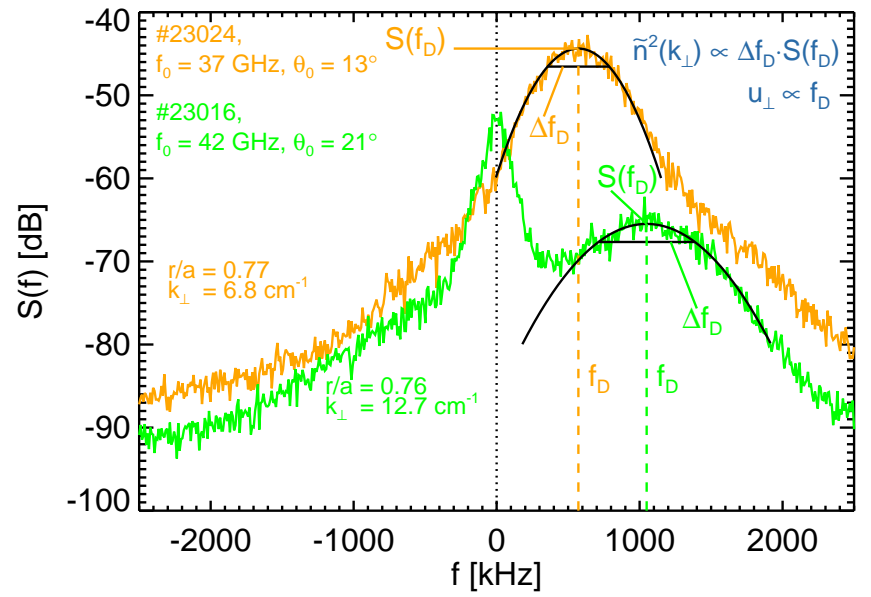

FIG. 3: (\#23016, \#23024) Example Doppler shifted spectra measured at different $k_{\perp}$. Since for higher $k_{\perp}$ the turbulence level decreases, the Doppler peak amplitude is lower.

ular attention was paid to the microwave beam shape in the plasma in order to optimize the system to the given requirements [50].

Figure 3 shows two Doppler shifted spectra measured at roughly the same radial position $(\rho=r / a=0.76$ and $0.77, a$ the minor plasma radius) from comparable neutral beam injection (NBI) heated plasmas in TJ-II. The Doppler peaks are at around 570 and $1050 \mathrm{kHz}$ for low and high $k_{\perp}$, respectively. As pointed out above, the Doppler shift $f_{\mathrm{D}}$ gives the perpendicular velocity of density fluctuations $u_{\perp}$ and the area under the Doppler peak reflects the density fluctuation level $\tilde{n}^{2}$, which is roughly two orders of magnitude lower for the higher $k_{\perp}$ case. To obtain the properties of the Doppler shifted peak, a Gaussian is fitted to the spectrum, which yields the Doppler shift $f_{\mathrm{D}}$, the width $\Delta f_{\mathrm{D}}$ and the amplitude $S\left(f_{\mathrm{D}}\right)$, as shown in Fig. 3. As pointed out above, $f_{\mathrm{D}} \propto u_{\perp}$ and $\Delta f_{\mathrm{D}} \cdot S\left(f_{\mathrm{D}}\right) \propto \tilde{n}^{2}$, which is proportional to the area under the Gaussian. The Doppler shifts in Fig. 3 correspond to velocities of $5.3\left(k_{\perp}=6.8 \mathrm{~cm}^{-1}\right)$ and $5.2 \mathrm{~km} / \mathrm{s}\left(k_{\perp}=12.7 \mathrm{~cm}^{-1}\right)$. The zero-order component centered around $f=0$ in the $k_{\perp}=12.7 \mathrm{~cm}^{-1}$ case is measured because for high $k_{\perp}$, the backscattered power is low and consequently a high fraction of the microwave beam power is reflected and reaches the vessel wall. After reflection at the wall, it returns to the plasma, is reflected again and goes back into the antenna. Since the Doppler peak amplitude is low for high $k_{\perp}$, this zeroorder component can become stronger in amplitude than the Doppler peak. However, since for high $k_{\perp}$ measured Doppler shifts are strong and thus the zero-order reflection and the Doppler peak are well separated, this does not cause a significant problem in the data analysis.

To localize the Doppler reflectometer measurement position $\rho$ and to infer the turbulence wavenumber $k_{\perp}$, the three-dimensional ray tracing code Truba [51] is used. To estimate uncertainties in the measurements, two ad- ditional rays are launched in parallel to the central ray, separated by one beam waist (where the beam power is a factor $1 / e^{2}$ of the central beam power). This method gives a turning point for each ray, yielding an estimate of the uncertainty in the radial localization of the measurement, which is added to the uncertainty in the AM reflectometer profile reconstruction $(\Delta \rho=0.01)$. The values of $k_{\perp}$ and $B$ at the individual ray turning points are then extracted and give the respective uncertainties, $\Delta k_{\perp}<2 \mathrm{~cm}^{-1}$ and $\Delta B<25 \mathrm{mT}$ for all measurements shown here. The uncertainty in the extraction of the Doppler shift is around $20 \mathrm{kHz}$. The resulting error in the radial electric field estimation is $\Delta E_{r}<1 \mathrm{kV} / \mathrm{m}$. The numerical extraction of the density fluctuation level is very robust with an estimated error $\Delta S\left(k_{\perp}\right)<1 \mathrm{~dB}$. However, since the power output of the microwave oscillator and the throughput of active microwave components are frequency-dependent, a careful calibration of a Doppler reflectometer is necessary in order to extract the signal power. To this end the launching and receiving waveguides were disconnected from the antenna and joined by a $180^{\circ}$ turn which included a variable attenuator to avoid system saturation. In this way, the emitted power is transferred directly into the receiving part of the system. The antenna and mirror frequency dependences are not considered with this method. However, the antenna directivity varies only by about 2.5 $\mathrm{dB}$ in the whole frequency range, which is small compared to the dynamic range of the system $(\sim 50 \mathrm{~dB})$. Furthermore, two dimensional full-wave simulations for the mirror show power losses of less than $1 \%$ at different frequencies and tilt angles. With the above calibration, for each microwave frequency a calibration factor is obtained, which is later used to normalize the measurement. From a physics point of view, two-dimensional full-wave simulations have shown that the wavenumber spectrum reconstruction is most reliable in the linear turbulence regime, while in the non-linear regime, turbulence levels could be slightly underestimated (few dB) [48]. In the context of the present work, the H-mode measurements show considerably lower turbulence levels than the Lmode measurements, hence the turbulence reduction in $\mathrm{H}$-mode is more likely to be even underestimated. Since trends in the turbulence reduction - which is more than $10 \mathrm{~dB}$ - are considered, this effect does not present a problem here.

\section{EXPERIMENTAL RESULTS}

Experiments were carried out in neutral beam injection $\left(\mathrm{NBI}, P_{\mathrm{NBI}}=370 \mathrm{~kW}\right)$ co-heated plasmas pre-heated by off-axis ECRH (two gyrotrons, $P_{\mathrm{ECRH}}=2 \times 230 \mathrm{~kW}$ ). Line-averaged densities were $\left\langle n_{e}\right\rangle_{\mathrm{L}}=2.3 \times 10^{19} \mathrm{~m}^{-3}$ and $\left\langle n_{e}\right\rangle_{\mathrm{H}}=2.7 \times 10^{19} \mathrm{~m}^{-3}$ for L- and H-mode, respectively. Electron temperatures $T_{e}$ in the measurement region of this paper were between 100 and $180 \mathrm{eV}$. A total of 33 comparable discharges were performed, during which 


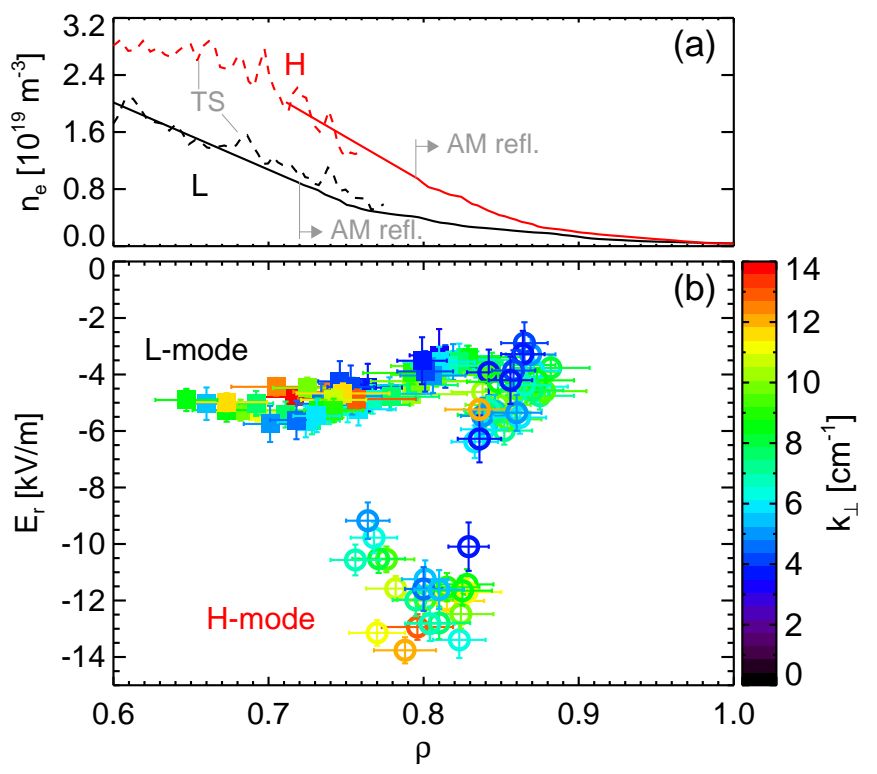

FIG. 4: (a) Density profiles in L- and H-mode measured by Thomson scattering (dashed) and AM reflectometry (solid). (b) Radial $E_{r}$ profiles in L- (squares) and $\mathrm{H}$-mode (circles). The $k_{\perp}$ value used to infer $E_{r}$ is color-coded.

both turbulence scale and radial measurement position of the Doppler reflectometer were varied on a shot-to-shot basis.

\section{A. Radial Electric Field in L- and H-mode}

Figure 4(a) shows density profiles measured during Land $\mathrm{H}$-mode phases of the discharges. Dashed lines indicate measurements by the Thomson scattering (TS) system [52] and solid lines are obtained from an AM reflectometer [53]. Since the AM reflectometer profiles are measured in the edge plasma (up to a cutoff density of $n_{e} \approx 10^{19} \mathrm{~m}^{-3}$ ), it is only used for the outer region. For the inner part of the profile, a linear extrapolation is used which is aligned to the Thomson scattering measurements. The resulting density profiles are used as input profiles for the ray tracing code Truba.

In Fig. 4(b), the radial $E_{r}$ profiles in L-mode (squares) and H-mode (circles) are shown. The turbulence scale $k_{\perp}$ measured to obtain $E_{r}=2 \pi f_{\mathrm{D}} B / k_{\perp}$ is color-coded. Note that the inferred $E_{r}$ does not depend on $k_{\perp}$. This can either mean that $v_{\text {ph }} \ll v_{E \times B}$ or that $v_{\text {ph }}$ does not depend on the value of $k_{\perp}$, conclusions of particular importance not only for Doppler reflectometry, but also for turbulence investigations. In general, in turbulence theory, $v_{\text {ph }}$ does depend on $k_{\perp}$. In drift wave turbulence the wavenumber dependence of the phase velocity is due to the polarization drift, in particular for small structures $k_{\perp} \rho_{\mathrm{S}}>1$. The above supports the conclusion that $v_{\mathrm{ph}} \ll v_{E \times B}$ in TJ-II, already seen for ECRH and NBI L-mode plasmas [35].
In L-mode, $E_{r}$ is rather flat with values around -4 to $-6 \mathrm{kV} / \mathrm{m}$. The measurement positions for the H-mode plasma are further outside due to the higher edge density. In $\mathrm{H}$-mode, the $E_{r}$ profile develops a pronounced gradient leading to a strong shear layer located at $\rho \approx 0.83$, which is important to the subject of this paper. From this position towards the edge plasma, the $E_{r}$ values are similar to those measured in L-mode. However, from the shear layer towards the center, a radial electric field strength of up to $-14 \mathrm{kV} / \mathrm{m}$ is obtained. Further inside $(\rho<0.78)$, $E_{r}$ starts to decrease again, hence a well is formed as observed in the transport barriers of both stellarators and tokamaks $[5,6,35,40,41,54,55]$.

The strong $E_{r}$ shear at $\rho \approx 0.83$ is not due to the pressure gradient, which shows no pronounced changes at this radial position. A possible explanation could be a large-scale flow driven by turbulence, which has recently been observed in several experiments, such as TJ-II [56], NSTX [57], ASDEX Upgrade [58] and DIII-D [59].

\section{B. Turbulence Spectra L- and H-mode}

For clarity, the $E_{r}$ profiles from Sec. III A are repeated in Figs. 5 for the L- (a) and H-mode (c) phases. The lower parts of the Figs. (b, d) indicate the available data in the $k_{\perp}-\rho$ space, both $k_{\perp}$ and $\rho$ are calculated from Truba. From this, four different radial regions can be used to estimate wavenumber spectra. In L-mode, the radial range is divided into four intervals of $\Delta \rho=0.05$, as indicated in Fig. 5(a) by the vertical dotted lines. This gives about 15 points for each $k_{\perp}$ spectrum, except for the inner one $(\rho=0.64-0.70)$, where seven measurements are available (see Fig. $5(\mathrm{~b})$ ). For fixed $\theta_{0}$, the probed perpendicular wavenumbers increase towards the plasma center, because $f_{0}$ (hence $k_{0}$ ) increases (cf eq. 1 ). An additional effect can be attributed to the fact that the angle of incidence of the microwave beam with the cutoff layer normal increases as the beam enters more to the plasma center. See Fig. 2(b) for an illustrative example. The tilt angle scan spans the wavenumber space in the other direction, as indicated by the arrows in the upper right corner. In the outer part of the measured region, wavenumbers from around 3 to $10 \mathrm{~cm}^{-1}$ are scanned, while further inside, the wavenumber range increases and is between 4 and $15 \mathrm{~cm}^{-1}$. The labels (a, b, c, d) at the bottom of the plot identify the wavenumber spectra plotted in Fig. 6.

In case of the H-mode, the $E_{r}$ profile must be classified in four different sections from a physics point of view. They are marked in Fig. $5(\mathrm{c}, \mathrm{d})$ by the vertical dotted lines and labeled as the edge region $(\rho=0.84-$ $0.89)$, the shear region with strong $E_{r}$ shear $(\rho=0.82-$ $0.84)$, the $E_{r}$ well region $(\rho=0.78-0.82)$, and finally the core region $(\rho=0.75-0.78)$, where $E_{r}$ starts to decrease towards the plasma center. As for L-mode, in the edge, the accessible $k_{\perp}$ values are slightly lower than further inside, resulting in wavenumber spectra for slightly 

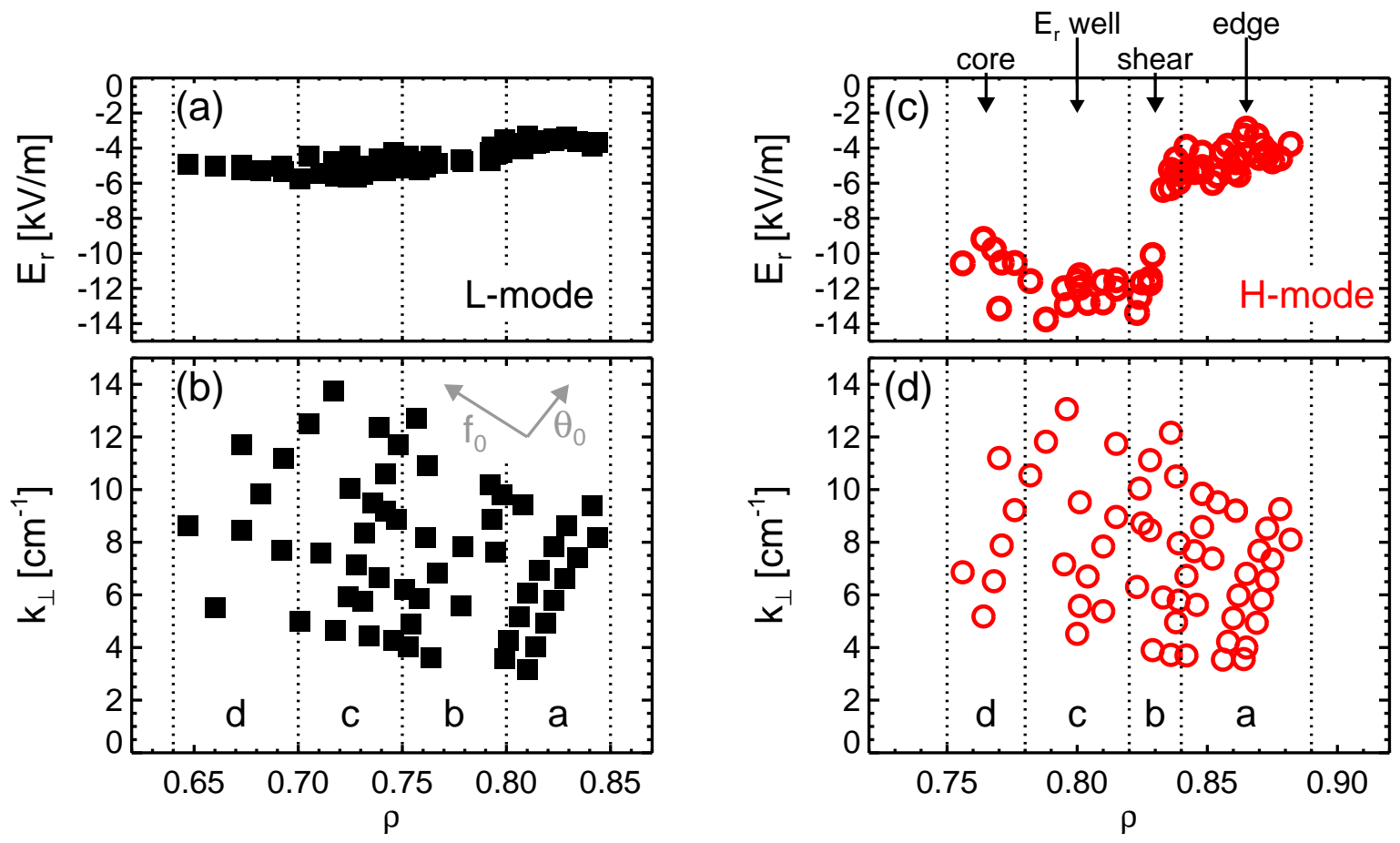

FIG. 5: (\#23013-51) Determination of radial regions where $k_{\perp}$-spectra are calculated. (a, c) $E_{r}$ profiles and (b, d) wavenumber space covered for L- and H-mode. For details refer to the text.

larger scales. For the innermost region only few points are available. The labels $(\mathrm{a}, \mathrm{b}, \mathrm{c}, \mathrm{d})$ at the bottom of the plot identify the wavenumber spectra plotted below in Fig. 7. For both $\mathrm{L}$ - and $\mathrm{H}$-modes the corresponding normalized wavenumbers are in the range $k_{\perp} \rho_{s} \approx 0.5-$ $2.5\left(\rho_{s}=0.1-0.2 \mathrm{~cm}\right)$, where $\rho_{s}=\sqrt{2 m_{i} T_{e}} / e B$ is the ion Larmor radius evaluated at electron temperature.

In Fig. 6, the L-mode wavenumber spectra for the radial regions defined in Figs. 5(a, b) are plotted. The horizontal dashed line at $-20 \mathrm{~dB}$ serves to guide the eye. In all plots, $k_{\perp} \rho_{s}=1$ is marked as a dotted vertical line and the $E_{r}$ profile is shown as an inset where the respective measurement region is indicated by a gray stripe. From (a) to (d), the probed region moves from the plasma edge towards the plasma center. A general observation is that from large towards small scales (all scales smaller than the system size, $k \approx 0.3 \mathrm{~m}^{-1}$ ) the density turbulence level decreases, which is observed also in 2D and 3D neutral fluid turbulence [60]. Furthermore, a power law behavior can be identified. Beginning with the outermost spectrum (a), there is a flat region from $k_{\perp}=3$ to $5 \mathrm{~cm}^{-1}$, where the spectral fall-off starts. The spectral index is $\alpha=-2.0 \pm 0.5$. Further inside ((b) and (c)), in the $k_{\perp}$ range between 4 and $9 \mathrm{~cm}^{-1}$, spectral indices with values of $\alpha=-1.8 \pm 0.2$ and $-2.4 \pm 0.4$ are found. A fall-off at higher $k_{\perp}$ at around $k_{\perp}=9 \mathrm{~cm}^{-1}$ becomes apparent. The spectral index of the high $k_{\perp}$ power law in (b) is $\alpha=-10.2 \pm 1.0$. Due to the lack of data in the $k_{\perp}$ spectrum (d), no conclusions are drawn apart from the spectral fall-off towards small scales.
In the H-mode (Fig. 7), the turbulence level decreases with decreasing structure size as in L-mode. In the edge $k_{\perp}$ spectrum (a), a power law with $\alpha=-2.6 \pm 0.3$ can be identified followed by a pronounced spectral fall-off towards higher $k_{\perp}$ with $\alpha$ between -9 and -13 (large data scatter). In the shear region (b), the spectral index is $\alpha=-3.1 \pm 0.8$, and the turbulence level has dropped by about one order of magnitude in comparison with the edge measurement and also in comparison with the Lmode spectra. The spectral index of the high $k_{\perp}$ region is $\alpha=-5.7 \pm 0.6$, which is lower than the high $k_{\perp}$ spectral index in L-mode (cf Fig. 6(b)), indicating that turbulence reduction is most effective at intermediate turbulence scales. In the $E_{r}$ well region (c), the turbulence level is also reduced (and lower than in L-mode). However, it increases slightly with respect to the shear layer measurement (b). The power law shows a spectral index of $\alpha=-2.4 \pm 0.5$, comparable to the edge measurement. Note the spectral index in the shear region is smaller than the one in both the edge and $E_{r}$ well regions in H-mode and also all the power laws in L-mode, hinting that not the largest, but intermediate turbulence scales are preferentially suppressed. Also note that in all spectra, except the one in the $E_{r}$ shear region, the knee-point is at around $k_{\perp} \approx 9 \mathrm{~cm}^{-1}$ and to the right of the $k_{\perp} \rho_{s}=1$ line. In the $E_{r}$ shear region, the knee is at $k_{\perp} \approx 6 \mathrm{~cm}^{-1}$ and to the left of $k_{\perp} \rho_{s}=1$, which could hint that turbulence characteristics have changed substantially at this radial position. For the core region (d), the turbulence level is higher than in all spectra towards the edge. 

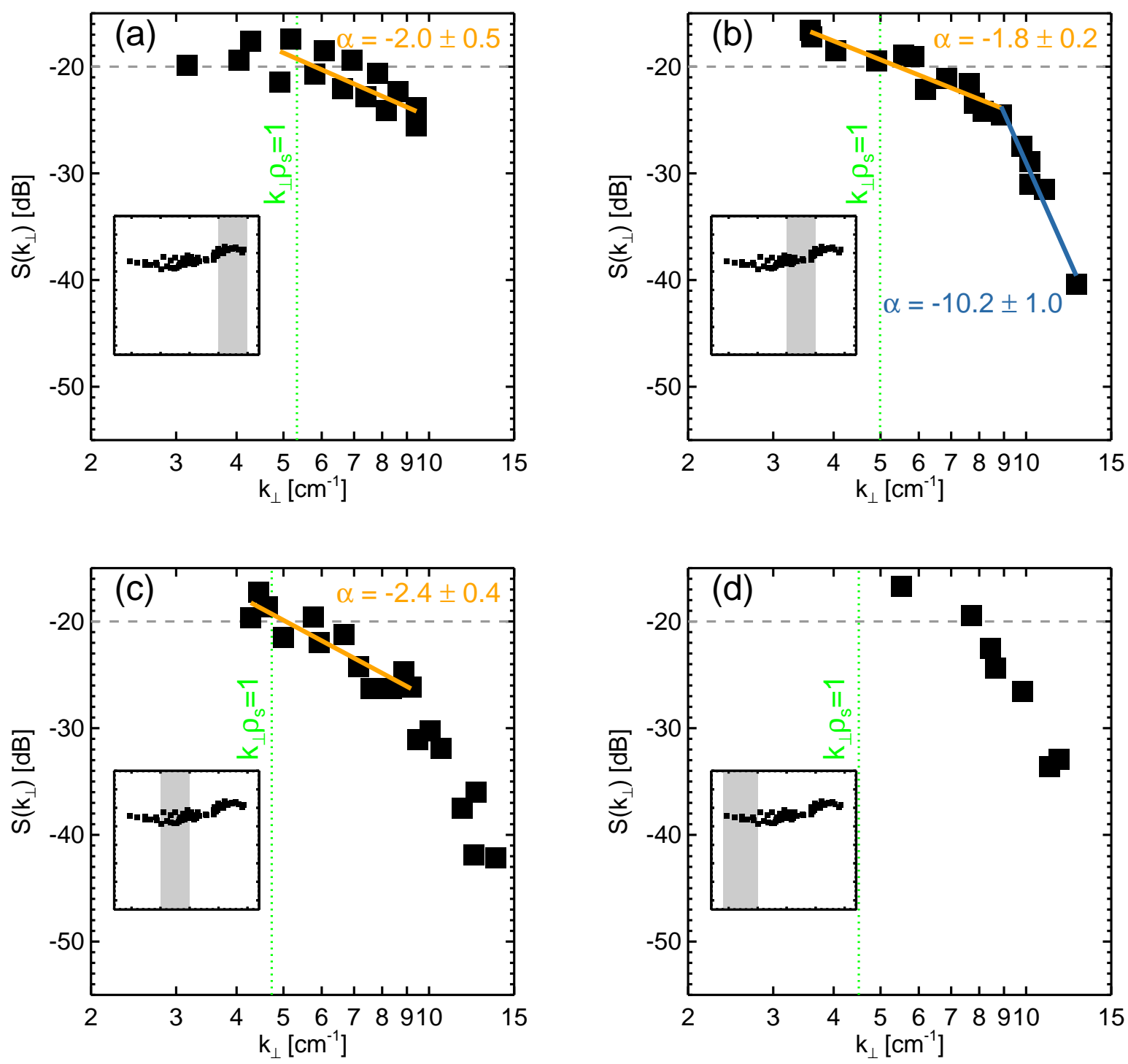

FIG. 6: (\#23013-51) L-mode wavenumber spectra for different radial regions indicated in the inset $E_{r}$ profile at the bottom left of each plot. The dashed line at $-20 \mathrm{~dB}$ serves to guide the eye. For details refer to the text.

These measurements compare well to existing literature: far-infrared measurements in a tokamak plasma (Mictrotor tokamak) were made in 1980 by Semet et al. and yielded $\alpha=-3.5$ in the range $6 \mathrm{~cm}^{-1}<k_{\perp}<20$ $\mathrm{cm}^{-1}$ [61]. Devynck et al. investigated $k_{\perp}$ spectra in 1993 in the Tore Supra tokamak with collective infrared laser scattering and obtained spectral indices $\alpha=-3$ at $k_{\perp}>6 \mathrm{~cm}^{-1}[24]$. These results were confirmed by Zou et al. through the first $k_{\perp}$ spectrum measurement by Doppler reflectometry in 1999 for the $k_{\perp}>4 \mathrm{~cm}^{-1}$ range, where $\alpha=-2.8$ was measured [39]. Hennequin et al. observed a stronger spectral index at high $k_{\perp}>15 \mathrm{~cm}^{-1}$, measurements recently extended by Vermare et al. by studying the collisionality dependence of the wavenumber spectra [27]. The measurements referred to have been obtained exclusively in L-mode plasmas. To our knowledge, no systematic comparison of $\mathrm{H}$-mode to L-mode wavenumber spectra has been published yet, although preliminary studies have been presented with spectral indices between -4 and -7 [28-30].

\section{Radial Fluctuation Profiles in L- and H-mode}

To calculate the wavenumber spectra above, the data were grouped into different radial regions, and the wavenumber spectrum was plotted for each region. Another representation is to group the measured data into different wavenumber ranges and plot the radial turbulence level dependence, shown in Fig. 8. In the L-mode data (a), the decrease of density fluctuation level towards smaller scales is visible. However, the measurements show no radial fluctuation level dependence. In contrast, in H-mode, a strong fluctuation level reduction 

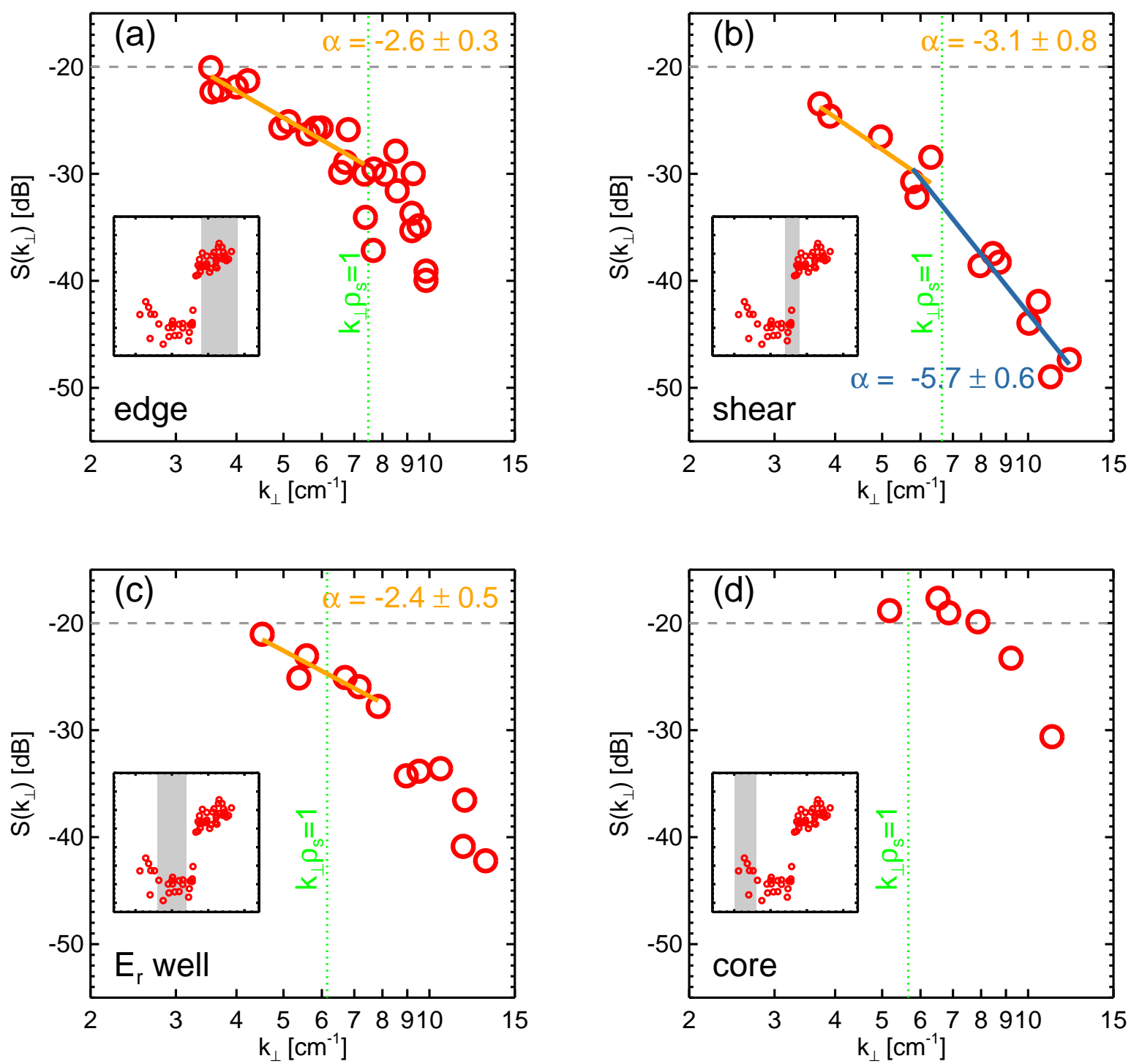

FIG. 7: (\#23013-51) Same as Fig. 6 for H-mode.

close to the $E_{r}$ shear layer can be observed, as already seen in Sec. III B. Furthermore, a stronger reduction of intermediate scale turbulence $\left(k_{\perp}=7-11 \mathrm{~cm}^{-1}\right.$, corresponding to $\left.k_{\perp} \rho_{s}=1.0-1.7\right)$ compared to the larger and smaller scales can be identified, which is visible in the more pronounced minima of turbulence level in Fig. 8(b). This confirms the conclusion obtained by interpreting the power laws of the wavenumber spectrum in the $E_{r}$ shear region (fig. $7(\mathrm{~b})$ ).

\section{DISCUSSION AND CONCLUSIONS}

The fact that turbulence energy in the whole wavenumber range is reduced in $\mathrm{H}$-mode with respect to the $\mathrm{L}$ mode plasma in the region of maximum $E_{r}$ shear (and not in the edge or at the innermost measurement posi- tion), is a strong hint that the $E_{r}$ shear and the resultant strong $E \times B$ shear flows play a central role in the reduction of the edge turbulence level in TJ-II H-mode plasmas.

If shear decorrelation of large structures were dominant, the radial correlation length of large-scale structures should be reduced, and the energy of the resulting smaller scales should be enhanced. This would result in more density turbulence energy at higher $k_{\perp}$. Then the power at high $k_{\perp}$ should increase while at low $k_{\perp}$ it should decrease. This is not observed here, so either the shear decorrelation mechanism does not play a central role or strongly non-isotropic structures are measured. However, anisotropic turbulence has been observed mainly at large scales $\left(k_{\perp}<2 \mathrm{~cm}^{-1}\right)$, while at smaller scales structures are isotropic [9, 19, 62, 63].

The turbulence reduction is observed to happen preferentially on intermediate scales. Through the sheared 


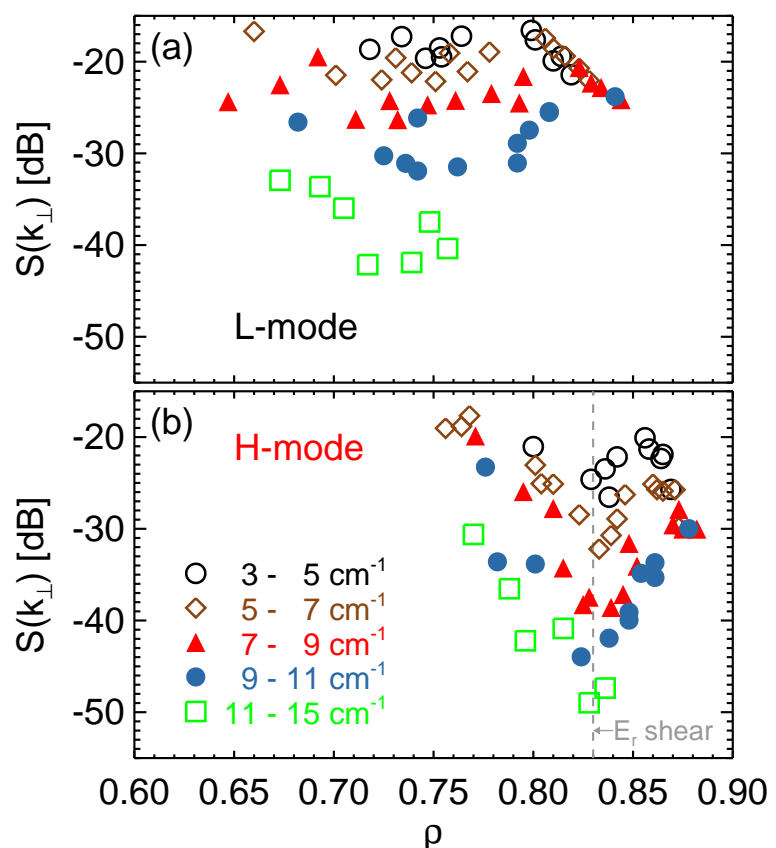

FIG. 8: (\#23013-51) Radial profiles of scale-resolved density fluctuation level in (a) L- and in (b) H-mode. In L-mode, the turbulence level is rather constant in the observed radial range. In $\mathrm{H}$-mode, at the $E_{r}$ shear location, the reduction is strongest. Of the scales investigated, the intermediate scales $\left(k_{\perp}=7-11 \mathrm{~cm}^{-1}\right)$ are reduced most effectively.

$E \times B$ flow the structures are tilted and thus the Reynolds stress becomes effective. The reduction of turbulence on all investigated scales points to an energy sink which is not provided by the shear decorrelation mechanism. A possible explanation of the reduction of spectral power is the transfer of energy. Large-scale structures might not be affected by the sheared flow due to their stronger vorticity [64]. In particular, both in simulations [13] and also in other experiments [12], it was found that the main contribution to $\mathrm{ZF}$ drive comes from smaller scales $k_{\theta} \rho_{s} \gtrsim 0.7$ and 1.0 , respectively. The preferential turbulence energy reduction for the present TJ-II measurements takes place on scales $k_{\perp} \rho_{s} \approx 1.0-1.7$, consistent with these previously observed results. The other mechanism, shear decorrelation of turbulent structures, might not play a central role here: neither are large scales preferably reduced, nor is the energy redistributed towards smaller scales. However, it cannot be excluded that both mechanisms are active here.

In summary, density fluctuation wavenumber spectra have been measured in L- and $\mathrm{H}$-mode plasmas of the TJ-II stellarator. The wavenumber spectra exhibit the typical shape with a plateau at low $k_{\perp}$ and a decay at higher $k_{\perp}$. The spectral indices in the L-mode lie between -1.8 and -2.4 . In $\mathrm{H}$-mode, power laws with $\alpha$ between -2.4 and -3.1 are found. Close to the $E_{r}$ shear layer, the turbulence energy is decreased, and this effect is most pronounced at intermediate scales (reduction by about one order of magnitude in comparison to L-mode). At smaller radii, where $E_{r}$ is strong but its shear moderate, the turbulence energy is reduced as well, but not as much as in the $E_{r}$ shear layer $(\rho=0.82-0.84)$. At even smaller radii, the turbulence energy is level with the Lmode measurement.

The preferential reduction of intermediate turbulence scales can be explained within the framework of vortex straining and subsequent energy transfer through Reynolds stress into zonal flows, as suggested in Refs. [12, 15]. It is questionable whether turbulence decorrelation by sheared flows plays a role in TJ-II H-mode plasmas, although it cannot be excluded that both mechanisms are active. Further studies, e.g. the inclusion of Doppler radial correlation reflectometry $[65,66]$, which measures $k_{r}$ at low turbulence amplitudes and strong velocity shear which is the case in $\mathrm{H}$-mode -, would yield information on the degree of anisotropy of turbulent eddies, as could fast camera measurements [67] and beam emission spectroscopy [9], which could thus contribute significantly to the measurements and help to clarify the physics mechanisms at work.

\section{Acknowledgments}

The authors gratefully acknowledge the TJ-II team for conducting the experiments. This work was partially funded by the Spanish Ministry of Science and Innovation under Contract No. ENE2010-18409, which is gratefully acknowledged.
[1] F. Wagner, G. Becker, K. Behringer, D. Campbell, A. Eberhagen, W. Engelhardt, G. Fussmann, O. Gehre, J. Gernhardt, G. v. Gierke, G. Haas, M. Huang, F. Karger, M. Keilhacker, O. Klber, M. Kornherr, K. Lackner, G. Lisitano, G. G. Lister, H. M. Mayer, D. Meisel, E. R. Mller, H. Murmann, H. Niedermeyer, W. Poschenrieder, H. Rapp, and H. Röhr, Phys. Rev. Lett. 49, 1408 (1982).

[2] R. J. Groebner, K. H. Burrell, and R. P. Seraydarian, Phys. Rev. Lett. 64, 3015 (1990).
[3] K. Ida, S. Hidekuma, Y. Miura, T. Fujita, M. Mori, K. Hoshino, N. Suzuki, T. Yamauchi, and J.-M. Group, Phys. Rev. Lett. 65, 1364 (1990).

[4] C. P. Ritz, H. Lin, T. L. Rhodes, and A. J. Wootton, Phys. Rev. Lett. 65, 2543 (1990).

[5] E. J. Doyle, R. J. Groebner, K. H. Burrell, P. Gohil, T. Lehecka, J. N. C. Luhmann, H. Matsumoto, T. H. Osborne, W. A. Peebles, and R. Philipona, Phys. Fluids B 3, 2300 (1991). 
[6] K. H. Burrell, T. N. Carlstrom, E. J. Doyle, D. Finkenthal, P. Gohil, R. J. Groebner, D. L. Hillis, J. Kim, H. Matsumoto, R. A. Moyer, T. H. Osborne, C. L. Rettig, W. A. Peebles, T. L. Rhodes, H. StJohn, R. D. Stambaugh, M. R. Wade, and J. G. Watkins, Plasma Phys. Control. Fusion 34, 1859 (1992).

[7] H. Biglari, P. H. Diamond, and P. W. Terry, Phys. Fluids 2, 1 (1990).

[8] P. W. Terry, Rev. Mod. Phys. 72, 109 (2000).

[9] G. R. McKee, R. J. Fonck, D. K. Gupta, D. J. Schlossberg, M. W. Shafer, R. L. Boivin, and W. Solomon, Plasma Fus. Res. 2, S1025 (2007).

[10] A. Fujisawa, K. Itoh, H. Iguchi, K. Matsuoka, S. Okamura, A. Shimizu, T. Minami, Y. Yoshimura, K. Nagaoka, C. Takahashi, M. Kojima, H. Nakano, S. Ohsima, S. Nishimura, M. Isobe, C. Suzuki, T. Akiyama, K. Ida, K. Toi, S.-I. Itoh, and P. H. Diamond, Phys. Rev. Lett. 93, 165002 (2004).

[11] P. H. Diamond, S.-I. Itoh, K. Itoh, and T. S. Hahm, Plasma Phys. Control. Fusion 47, R35 (2005).

[12] P. Manz, M. Ramisch, and U. Stroth, Phys. Rev. Lett. 103, 165004 (2009).

[13] B. D. Scott, New J. Phys. 7, 92 (2005).

[14] V. Naulin, New J. Phys. 4, 28 (2002).

[15] U. Stroth, P. Manz, and M. Ramisch, Plasma Phys. Control. Fusion 53, 024006 (2011).

[16] C. Holland and P. H. Diamond, Phys. Plasmas 11, 1043 (2004).

[17] R. E. Waltz, J. Candy, and M. Fahey, Phys. Plasmas 14, 056116 (2007).

[18] E. Mazzucato, Phys. Rev. Lett. 36, 792 (1976).

[19] C. M. Surko and R. E. Slusher, Phys. Rev. Lett. 37, 1747 (1976).

[20] E. Mazzucato, Phys. Rev. Lett. 48, 1828 (1982).

[21] T. Crowley and E. Mazzucato, Nucl. Fusion 25, 507 (1985).

[22] D. Brower, W. Peebles, and N. L. Jr., Nucl. Fusion 27, 2055 (1987).

[23] G. McKee, C. Petty, R. Waltz, C. Fenzi, R. Fonck, J. Kinsey, T. Luce, K. Burrell, D. Baker, E. Doyle, X. Garbet, R. Moyer, C. Rettig, T. Rhodes, D. Ross, G. Staebler, R. Sydora, and M. Wade, Nucl. Fusion 41, 1235 (2001).

[24] P. Devynck, X. Garbet, C. Laviron, J. Payan, S. K. Saha, F. Gervais, P. Hennequin, A. Quéméneur, and A. Truc, Plasma Phys. Control. Fusion 35, 63 (1993).

[25] P. Hennequin, R. Sabot, C. Honoré, G. T. Hoang, X. Garbet, A. Truc, C. Fenzi, and A. Quéméneur, Plasma Phys. Control. Fusion 46, B121 (2004).

[26] P. Hennequin, C. Honoré, A. Truc, A. Quéméneur, C. Fenzi-Bonizec, C. Bourdelle, X. Garbet, and G. T. Hoang, Nucl. Fusion 46, S771 (2006).

[27] L. Vermare, P. Hennequin, O. D. Gurcan, C. Bourdelle, F. Clairet, X. Garbet, R. Sabot, and the Tore Supra Team, Phys. Plasmas 18, 012306 (2011).

[28] G. D. Conway, C. Tröster, J. Schirmer, C. Lechte, W. Suttrop, E. Poli, H. Zohm, and A. U. Team, Turbulence measurements using Doppler reflectometry on ASDEX Upgrade (Proc. $9^{\text {th }}$ International Reflectometry Workshop, Lisbon, Portugal, 2009).

[29] T. Happel, T. Estrada, G. D. Conway, E. Blanco, T.-I. Team, and A. U. Team, Scale-resolved Turbulence Studies in L- and H-mode Plasmas of TJ-II and ASDEX Upgrade (Proc. $37^{\text {th }}$ EPS Conference on Plasma Physics, Dublin, Ireland, 2010), ECA Vol. 34A, P1.1039.
[30] T. Happel, T. Estrada, E. Blanco, G. D. Conway, W. Kasparek, B. Plaum, C. Lechte, D. Wagner, U. Stroth, T.-I. Team, and A. U. Team, Scale-selective Turbulence Reduction in H-mode Plasmas in the TJ-II Stellarator \& Design of a new Doppler Reflectometer Front End for the ASDEX Upgrade Tokamak $\left(10^{\text {th }}\right.$ International Reflectometry Workshop, Padua, Italy, 2011).

[31] E. Z. Gusakov, A. D. Gurchenko, A. B. Altukhov, V. V. Bulanin, L. A. Esipov, M. Y. Kantor, D. V. Kouprienko, S. I. Lashkul, A. V. Petrov, and A. Y. Stepanov, Plasma Phys. Control. Fusion 48, B443 (2006).

[32] T. L. Rhodes, W. A. Peebles, J. C. DeBoo, R. Prater, J. E. Kinsey, G. M. Staebler, J. Candy, M. E. Austin, R. V. Bravenec, K. H. Burrell, J. S. deGrassie, E. J. Doyle, P. Gohil, C. M. Greenfield, R. J. Groebner, J. Lohr, M. A. Makowski, X. V. Nguyen, C. C. Petty, W. M. Solomon, H. E. S. John, M. A. V. Zeeland, G. Wang, and L. Zeng, Plasma Phys. Control. Fusion 49, B183 (2007).

[33] C. Alejaldre, J. J. A. Gozalo, J. B. Perez, F. C. Magana, J. R. C. Diaz, J. G. Perez, A. Lopez-Fraguas, L. Garcia, V. I. Krivenski, R. Martin, A. P. Navarro, A. Perea, A. Rodriguez-Yunta, M. S. Ayza, and A. Varias, Fusion Technol. 17, 131 (1990).

[34] T. Estrada, C. Hidalgo, and T. Happel, Nucl. Fusion 51, 032001 (2011).

[35] T. Estrada, T. Happel, L. Eliseev, D. López-Bruna, E. Ascasíbar, E. Blanco, L. Cupido, J. M. Fontdecaba, C. Hidalgo, R. Jiménez-Gómez, L. Krupnik, M. Liniers, M. E. Manso, K. J. McCarthy, F. Medina, A. Melnikov, B. v. Milligen, M. A. Ochando, I. Pastor, M. A. Pedrosa, F. L. Tabarés, D. Tafalla, and T.-I. Team, Plasma Phys. Control. Fusion 51, 124015 (2009).

[36] C. Hidalgo, M. A. Pedrosa, C. Silva, D. Carralero, E. Ascasibar, B. A. Carreras, T. Estrada, F. Tabars, D. Tafalla, J. Guasp, M. Liniers, A. Lpez-Fraguas, B. van Milligen, and M. A. Ochando, Europhys. Lett. 87, 55002 (2009).

[37] T. Estrada, T. Happel, C. Hidalgo, E. Ascasíbar, and E. Blanco, Europhys. Lett. 92, 35001 (2010).

[38] M. Hirsch, E. Holzhauer, J. Baldzuhn, and B. Kurzan, Doppler Reflectometry for the Investigation of poloidally propagating Density Perturbations (Proc. $4^{\text {th }}$ International Reflectometry Workshop, Cadarache, France, 1999), report EUR-CEA-FC-1674.

[39] X. L. Zou, T. F. Seak, M. Paume, J. M. Chareau, C. Bottereau, and G. Leclert, Poloidal Rotation Measurement in Tore Supra by Oblique Reflectometry (Proc. $4^{\text {th }}$ International Reflectometry Workshop, Cadarache, France, 1999), report EUR-CEA-FC-1674.

[40] M. Hirsch, E. Holzhauer, J. Baldzuhn, B. Kurzan, and B. Scott, Plasma Phys. Control. Fusion 43, 1641 (2001).

[41] G. D. Conway, J. Schirmer, S. Klenge, W. Suttrop, and E. Hozhauer, Plasma Phys. Control. Fusion 46, 951 (2004).

[42] P. Hennequin, C. Honoré, A. Truc, A. Quéméneur, N. Lemoine, J. M. Chareau, and R. Sabot, Rev. Sci. Instrum. 75, 3881 (2004).

[43] T. Happel, T. Estrada, E. Blanco, V. Tribaldos, A. Cappa, and A. Bustos, Rev. Sci. Instrum. 80, 073502 (2009).

[44] J. C. Hillesheim, W. A. Peebles, T. L. Rhodes, L. Schmitz, T. A. Carter, P.-A. Gourdain, and G. Wang, Rev. Sci. Instrum. 80, 083507 (2009).

[45] H.W. Müller, J. Adamek, R. Cavazzana, G. D. Conway, C. Fuchs, J. P. Gunn, A. Herrmann, J. Horacek, 
C. Ionita, A. Kallenbach, M. Kocan, M. Maraschek, C. Maszl, F. Mehlmann, B. Nold, M. Peterka, V. Rohde, J. Schweinzer, R. Schrittwieser, N. Vianello, E. Wolfrum, M. Zuin, and the ASDEX Upgrade Team, Nucl. Fusion 51, 073023 (2011).

[46] E. Blanco, T. Estrada, and J. Sánchez, Plasma Phys. Control. Fusion 48, 699 (2006).

[47] V. Bulanin and M. Yafanov, Plasma Phys. Rep. 32, 47 (2006).

[48] E. Blanco and T. Estrada, Plasma Phys. Control. Fusion 50, 095011 (2008).

[49] C. Lechte, IEEE Trans. Plasma Sci. 37, 1099 (2009).

[50] T. Happel, E. Blanco, and T. Estrada, Rev. Sci. Instrum. 81, 10D901 (2010).

[51] M. A. Tereshchenko, K. A. Sarksian, G. M. Batanov, A. S. Sakharov, R. Martín, F. Castejón, A. Fernández, and A. Cappa, Development and Use of 3D Ray/Beam Tracing Code for Plasma Heating by EBW in the TJ-II Stellarator (Proc. 30 ${ }^{\text {th }}$ EPS Conference on Contr. Fusion and Plasma Phys., St. Petersburg, Russia, 2003), Vol. 27A, p-1.18.

[52] C. J. Barth, F. J. Pijper, H. J. v. d. Meiden, J. Herranz, and I. Pastor, Rev. Sci. Instrum. 70, 763 (1999).

[53] T. Estrada, J. Sanchez, B. van Milligen, L. Cupido, A. Silva, M. E. Manso, and V. Zhuravlev, Plasma Phys. Control. Fusion 43, 1535 (2001).

[54] P. Gohil, K. Burrell, and T. Carlstrom, Nucl. Fusion 38, 93 (1998).

[55] R. M. McDermott, B. Lipschultz, J. W. Hughes, P. J. Catto, A. E. Hubbard, I. H. Hutchinson, R. S. Granetz, M. Greenwald, B. LaBombard, K. Marr, M. L. Reinke, J. E. Rice, D. Whyte, and A. C.-M. Team, Phys. Plasmas 16, 056103 (2009)

[56] T. Estrada, E. Ascasbar, T. Happel, C. Hidalgo, E. Blanco, R. Jimnez-Gmez, M. Liniers, D. Lpez-Bruna, F. Tabars, and D. Tafalla, Contrib. Plasma Phys. 50, 501 (2010).
[57] S. J. Zweben, R. J. Maqueda, R. Hager, K. Hallatschek, S. M. Kaye, T. Munsat, F. M. Poli, A. L. Roquemore, Y. Sechrest, and D. P. Stotler, Phys. Plasmas 17, 102502 (2010).

[58] G. D. Conway, C. Angioni, F. Ryter, P. Sauter, J. Vicente, and A. U. Team, Phys. Rev. Lett. 106, 065001 (2011).

[59] L. Schmitz, T. L. Rhodes, L. Zeng, E. J. Doyle, R. J. Groebner, J. C. Hillesheim, W. A. Peebles, K. H. Burrell, and G. Wang, Shear Flow and Turbulence Suppression in Limit Cycle Oscillations Preceding the L-H Transition (Joint US-EU Transport Taskforce Workshop, San Diego, USA, 2011).

[60] M. G. Shats, H. Xia, and H. Punzmann, Phys. Rev. E 71, 046409 (2005).

[61] A. Semet, A. Mase, W. A. Peebles, N. C. Luhmann, and S. Zweben, Phys. Rev. Lett. 45, 445 (1980).

[62] R. J. Fonck, N. Bretz, G. Cosby, R. Durst, E. Mazzucato, R. Nazikian, S. Paul, S. Scott, W. Tang, and M. Zarnstorff, Plasma Phys. Control. Fusion 34, 1993 (1992).

[63] M. Ramisch, N. Mahdizadeh, U. Stroth, F. Greiner, C. Lechte, and K. Rahbarnia, Phys. Plasmas 12, 032504 (2005).

[64] D. L. Eggleston, Phys. Plasmas 1, 3850 (1994).

[65] J. Schirmer, G. D. Conway, E. Holzhauer, W. Suttrop, H. Zohm, and the ASDEX Upgrade Team, Plasma Phys. Control. Fusion 49, 1019 (2007).

[66] E. Z. Gusakov and A. Popov, Theoretical analysis of radial correlation Doppler reflectometry $\left(10^{\text {th }}\right.$ International Reflectometry Workshop, Padua, Italy, 2011).

[67] J. A. Alonso, S. J. Zweben, P. Carvalho, J. L. de Pablos, E. de la Cal, C. Hidalgo, T. Klinger, B. P. van Milligen, R. J. Maqueda, M. A. Pedrosa, C. Silva, M. Spolaore, H. Thomsen, and the TJ-II team, Plasma Phys. Control. Fusion 48, B465 (2006). 